Strong correlation of elastin deletions, detected by FISH, with Williams syndrome: evaluation of 235 patients

Lowery MC, Morris CA, Ewart A, et al. Am f Hum Genet 1995;57:49-53.

Williams syndrome (WS) is recognised clinically when patients present with a variety of features including a characteristic dysmorphic appearance, supravalvular aortic stenosis (SVAS), mental deficiency, gregarious personality with "cocktail party" conversation, and idiopathic infantile hypercalcaemia. Patients with WS have a submicroscopic deletion at $7 \mathrm{q} 11.23$, detectable by FISH, which causes allelic loss of elastin (ELN). The authors used a weighted scoring system to classify patients:

Phenotypic feature

Typical facial features

Mental retardation/developmental delay

SVAS

Non-SVAS congenital heart disease Inguinal hernia

Hypercalcaemia

Total

Patients with 4 to 10 points were classified as "classic" cases of WS, and patients with 0 to 3 points as "uncertain". Ninety-six percent of patients with "classic" WS $(n=114)$ had molecular cytogenetic deletions, whereas only $8 \%(n=39)$ of "uncertain" WS had a deletion. A further 42 cases analysed were not classified phenotypically, and $60 \%$ of these had a deletion. The final 40 cases studied were referred to the clinical cytogenetics laboratory to "rule out WS", and 38\% of these had an ELN deletion, but no cytogenetic deletion on banded analysis. These results suggest that FISH can be very useful in providing confirmation of a clinical diagnosis, but the clinical scoring system used seems to have been very effective. The absence of a deletion on FISH analysis might indicate the need for clinical re-evaluation. One unresolved question at the moment is the question of whether or not the parental chromosomes should be routinely studied once a $7 \mathrm{q}$ deletion has been detected in a child. The recurrence risk for WS is very small, but experience from other microdeletion syndromes would suggest that parents can occasionally carry a translocation, or even be asymptomatic carriers of the same deletion. Perhaps with WS any sib pairs which do occur are more likely to be the result of germinal mosaicism.

FRANCES FLINTER

Multiple sites of anterior neural tube closure in humans: evidence from anterior neural tube defects (anencephaly) Golden JA, Chernoff GF. Pediatrics 1995;95: 506-10.

This study of anterior neural tube defects tested the hypothesis that humans, like mice and other experimental animals, have multiple sites of neural tube closure rather than the continuous "zipper-like" model of neural tube closure described in most embryology textbooks. Twenty fetuses and neonates with partial anencephaly (complete anencephaly would not provide the necessary information about closure sites) were ascertained and the rostral and caudal extents of the open neural tube defect were mapped onto a model cranium. The location of frequent closure defects could be observed directly as a result of the mapping process. Four closure sites for the anterior neural tube were identified and these corresponded closely to the four sites which have been described in the mouse. Mouse studies suggest that the time period during which these multiple closures can be observed in mouse embryos is very short. This may be why the observations have not been made previously in humans although Van Allen $e$ al (Am F Med Genet 1993; 47: 723-43) have also reported on this phenomenon. Neural tube defects are common malformations and studies suggest that they are multifactorial in origin, having both a genetic and an environmental contribution. Known environmental agents which can influence neural tube closure include folic acid and valproic acid. This study suggests that two or more different mechanisms could give rise to neural tube defects, the first being failure of two closures to meet and the second failure of a single closure. There is certainly genetic variation in the pattern of neural tube closure as evidenced by inbred strains of mice with differing susceptibilities and the particular type of neural tube defect seen in single gene disorders such as the Walker-Warburg syndrome. Some environmental factors also influence one closure site preferentially, for example, infants with fetal valproate syndrome tend to have very low lumbar or spinal defects. Correlation of the site of neural tube defects with particular aetiologies in this way, and clarification of the underlying mechanisms, could significantly influence counselling for recurrence risks and further our knowledge of possible modes of prevention.

JILL CLAYTON-SMITH

Apoptosis and loss of renal tissue in polycystic kidney diseases

Woo D. N Engl f Med 1995;333:18-25.

Polycystic kidney disease-there goes the neighbourhood

Grantham JJ. N Engl f Med 1995;333:56-7.

The inherited polycystic kidney diseases are characterised by the enlargement of renal cysts, interstitial fibrosis, and gradual loss of normal renal tissue. In autosomal dominant polycystic kidney disease, cysts originate in less than 1 to $2 \%$ of nephrons. David Woo, noting that renal insufficiency would be surprising if the many non-cystic nephrons remained functional despite the presence of neighbouring cysts, evaluated the possibility that non-cystic renal tubules in polycystic kidneys may be lost by apoptosis. Specific labelling of nuclear DNA fragmentation, a modification of the homopolymer tailing method, was used to allow in situ detection of apoptotic nuclei. Agarose gel electrophoresis was used to search for DNA ladders of oligo- nucleosomes arising from cleavage of chromatin before cell death. Both methods showed widespread apoptosis in both cystic and non-cystic nephrons of kidneys from people with autosomal recessive and autosomal dominant polycystic kidney disease as well as in the kidneys of $p c y$ and $c p k$ mice. In contrast, no evidence of apoptosis was detected in either normal controls or in specimens from patients with non-cystic renal diseases. The capacity of polycystic kidney cells to undergo apoptosis was retained in vitro. This study shows for the first time that apoptosis is an important mechanism in the pathogenesis of polycystic diseases. In his commentary, Grantham discusses this previously unsuspected process in inherited renal cystic diseases and points out that its recognition opens up another avenue of exploration for potential therapeutic opportunities. It is a new and interesting observation in a group of genetic disorders that cause significant morbidity and premature mortality in the population. It also comes at a time when, after many years of searching, the PKD1 gene and its product polycystin have finally been fully characterised.

\section{DAVID RAVINE}

\section{A single ataxia telangiectasia gene with} a product similar to P1-3 kinase

Savitsky K, Bar-Shira A, Gilad S, et al. Science 1995;268:1749-53.

It may at first seem surprising that the isolation of the gene for an obscure recessive disorder with an incidence of as little as 1 in 100000 is the cause of enormous interest. Ataxia telangiectasia (AT) is, however, a severe condition with cerebellar degeneration, immune deficiency, and death frequently before the age of 20 . In addition, sensitivity to radiation and increased risk of cancer are found not only in homozygotes but also among heterozygotes who constitute about $1 \%$ of the population and may therefore be one of the most numerous groups with an inherited predisposition to cancer. For instance, female heterozygotes have five times the risk of cancer than their normal counterparts. Having mapped the AT gene to a single contig from $11 \mathrm{q}$, this consortium have gone on to use cosmid and YAC clones for exon amplification of possible coding sequences and hybrid selection of complementary DNA sequences from cDNA libraries. From these, a transcription map of the area was produced and a single cDNA clone containing half the gene sequenced. This clone identified a major $12 \mathrm{~kb}$ evolutionarily conserved transcript in all tissues examined. Frameshift mutations have subsequently been identified in 14 patients, eight of whom are homozygous and six compound heterozygotes. The protein sequence is a mosaic of homologies to genes with potentially relevant functions including cell cycle control, DNA repair, and the coupling of the two. Strongest similarity is to the mammalian phosphatidylinositol-3' kinase (PI-3 kinase) gene which is part of a pathway thought to be involved in maintaining brain cells and 